(A257) Enhancing Human Resources for Health in Crisis: Experience from the War-Affected Districts of Sri Lanka K. Wickramage, ${ }^{1}$ T. Ranasinghe, ${ }^{2}$ A. Zwi ${ }^{3}$

1. Health Unit, Colombo, Sri Lanka

2. Government of Sri Lanka, Colombo, Sri Lanka

3. Faculty of Medicine, Sydney, Australia

Aims: This paper examines the coordination framework and interventions undertaken by the Sri Lankan health sector in providing essential health services to the displaced communities in the aftermath of the violent conflict in 2009. The narrative describes, in a chronological format, the strategies and actions undertaken by the health sector in response to a rapidly changing humanitarian crisis. The paper examines some of the key challenges faced by the health sector in the post-conflict recovery phases, the most pertinent being the human resources for health needs.

Methods: A review of Ministry of Health departmental meeting minutes/circulars, inter-agency health coordination meeting reports, weekly surveillance reports, inter-agency/agency assessments, media files, and donor and health cluster member reports were compiled and then analyzed in order to construct a narrative on how the Health Sector responded to the humanitarian crisis (from acute emergency phase to the post-conflict recovery and resettlement phase). The authors also were integrally involved in the planning, development, implementation, and monitoring of a spectrum of health sector interventions during the humanitarian crisis from within Government and the United Nations system.

Recommendations: A health systems strengthening approach, which places emphasis on human resources for health, can be effective in delivering high impact, sustained, high quality health care even in difficult and complex humanitarian emergencies such as civil war. The Sri Lankan experience has shown that harnessing effective human resource management stratergies in crisis also is vital for the post-conflict health system recovery phase that follows. The excuse that "the system may be too overwhelmed"; or health departments "too overstretched" to lead a coordinated effort can be mitigated with positive leadership and planning. The dividends of working in such an approach also ushers reconcilliation via a unified health workforce and promoting the idea of health as a bridge for peace.

Prehosp Disaster Med 2011;26(Suppl. 1):s71 doi:10.1017/S1049023X1100241X

(A258) Is It S.M.A.R.T. To S.T.A.R.T. To S.A.L.T. M.A.S.S. Casualty Victims?

R.L.Alson

Emergency Medicine, Winston-Salem, NC, United States of America

Triage of disaster and trauma victims is challenging, especially when responders have limited resources and brief periods of time. Over-triage of victims results in the consumption of resources that would be better utilized on more critical patients, and under-triage can result in increased mortality and/or morbidity, as victims do not receive the appropriate care. In addition, the same patient may be triaged multiple times as they move through echelons of care. These different echelons may have different objectives in the triage process Over the years, multiple triage schemes have been proposed and used, both in exercises and real events. None of these schemes is based on well-defined research, due to the difficulty of carrying out a randomized control study in real events. There has been a concerted effort to apply research findings in a effort to more effectively use resources and thus, improve patient outcomes as well as apply information garnered from after action reports. This presentation reviews the current issues and state of triage for disasters and mass-casualty incidents, drawing on examples from prior events. The ultimate objective of this presentation is to help the responder to better understand the process of triage and apply it to their clinical practice, thereby delivering care in an effective and timely manner.

Prehosp Disaster Med 2011;26(Suppl. 1):s71 doi:10.1017/S1049023X11002421

(A259) Objective Triage in the Disaster Setting: Will Children and Expecting Mothers be Triaged Like Others? T. Kouliev

Emergency Department, Beijing, China

Introduction: The study of disaster triage is made difficult by the complex emotional response of potentially lifesaving intervention that a triage officer must make decisions based on a succinct and efficient algorithm.

Methods: We designed a survey of triage professionals in Chicago, Philadelphia, and Beijing to identify sources of emotional bias that lead to failure of the START triage protocol that result in a lack of correlation between triage priority and clinical outcomes.

Results and Conclusions: Among our subjects, we observed that a pediatric victim is uniformly overtriaged when compared to less injured victims. We examine the possible reasons behind the consistency of this selection, explain the means we used to minimise bias, and propose avenues for further research and clinical implementation of better triage systems and guidelines.

Prehosp Disaster Med 2011;26(Suppl. 1):s71 doi:10.1017/S1049023X11002433

\section{(A260) Triage Decision-Making in Intoxication} A. Mirhaghi, ${ }^{\text {I }}$ G.R. Mohammadi, ${ }^{2}$ M. Asghari ${ }^{3}$

1. School of Nursing, Mashhad, Iran

2. School of Nursing, Neishabour, Iran

3. School Of Nursing, Gonabad, Iran

Background and Aims: Decision-making is the major component in triaging EDs patients. EDs Triage systems have applied different approaches to triaging intoxicated patients. Pros \& Cons for these approaches need to be identified. Aim is to analysis management of intoxicated patients during various triage process.

Methods: Critical review includes five triage systems, Emergency Severity Index, Australasian Triage Scale, Canadian triage and Acuity Scale, Manchester Triage System and 5-tier Triage protocol. These systems have been analyzed via meta-synthesis in terms of evidence-based criteria, inclusiveness, specific application and practicability.

Results: General physiologic signs \& symptoms were the gold standard for determining acuity in patients that have been applied by all triage systems. Conscious level, air way, respiratory status and circulation assessment were identified as major criteria in decision-making. 5-tier Triage protocol showed the 